\title{
Thermal quarks and gluon propagators in two-color dense QCD
}

\author{
Toru Kojo ${ }^{1, *}$ and Daiki Suenaga ${ }^{2, \uparrow}$ \\ ${ }^{1}$ Key Laboratory of Quark and Lepton Physics (MOE) and Institute of Particle Physics, \\ Central China Normal University, Wuhan 430079, China \\ ${ }^{2}$ Research Center for Nuclear Physics, Osaka University, Ibaraki 567-0048, Japan
}

(Received 16 February 2021; accepted 21 April 2021; published 12 May 2021)

\begin{abstract}
We study Landau gauge gluon propagators in two-color QCD at a finite quark chemical potential $\left(\mu_{q}\right)$ and temperature $(T)$. We include medium polarization effects at the one loop by quarks into massive gluon propagators and compare the analytic results with the available lattice data. We particularly focus on the high density phase of color-singlet diquark condensates whose critical temperature is $\sim 100 \mathrm{MeV}$ with a weak dependence on $\mu_{q}$. At zero temperature, the color singlet condensates protect the IR limit of electric and magnetic gluon propagators from the medium screening effects. At a finite temperature, this behavior remains true for the magnetic sector, but the electric screening mass should be generated by thermal, and hence gapless, particles which are unbound from the diquark condensates. Treating thermal excitations as quasiquarks, we found that the electric screening develops too fast as compared to the lattice results. Beyond the critical temperature for diquark condensates, the analytic results are consistent with the lattice results.
\end{abstract}

DOI: 10.1103/PhysRevD.103.094008

\section{INTRODUCTION}

Recently, there have been growing interests on the dynamics at baryon density ranging from $\sim 5 n_{0}$ to $\sim 40 n_{0}$ in the context of neutron star physics [1-5]. In this domain, baryons are supposed to overlap, and hence, quarks and gluons should be natural degrees of freedom $[6,7]$, while the matter is likely to be strongly correlated as one can infer from the breakdown of the perturbative calculations around $\sim 40 n_{0}[8,9]$. As the degrees of freedom are rather clear-cut, it is reasonable to expect the existence of some resummation with which strong $\alpha_{s}$ effects can be absorbed into the parameters of quasiparticles, e.g., effective mass, effective residues, so on, and after which residual interactions should become under control.

Along this line of thought, recently, we began to study quasiparticle descriptions of quarks and gluons in very dense matter [10,11]. Our descriptions are based on the Landau gauge QCD, which has been most extensively studied in functional methods [12-16] and lattice gauge theories at a zero baryon density $[17,18]$. In this gauge, the gluon propagators in the IR are tempered, and with a

\footnotetext{
torujj@mail.ccnu.edu.cn

'suenaga@rcnp.osaka-u.ac.jp
}

Published by the American Physical Society under the terms of the Creative Commons Attribution 4.0 International license. Further distribution of this work must maintain attribution to the author(s) and the published article's title, journal citation, and DOI. Funded by SCOAP. sufficiently large gluon mass, the infrared divergences associated with the perturbative running coupling constant can be avoided. Especially in the decoupling solution, which has been favored in lattice calculations, a gluon acquires the mass, $m_{g} \sim 500 \mathrm{MeV}$. These findings can be efficiently captured in the massive extension of the YangMills theory in the Landau gauge [19-24]. We expect that the presence of this mass makes our Feynman graph calculations better organized, sharpening our questions on the genuinely nonperturbative effects.

This point of view must be tested for finite density calculations. The two-color QCD is suitable for this purpose $[25,26]$, as this system allows us to perform lattice Monte Carlo simulations at a finite chemical potential [27-48]. The phase structure was analyzed by several analytic or continuum methods [49-57]. While at a low density, a dilute matter in two-color QCD is very different from the three-color QCD as the baryons in the former are bosons, at high densities, baryons overlap and the quark Fermi sea will be anyway established. It is this regime where we try to test our conjecture on the quasiparticle descriptions for the application to three-color QCD.

In the previous papers, we have studied gluon propagators with [11] and without [58] gluon masses by including the medium quark loops in the gluon polarizations. The calculations were performed at zero temperature in the presence of the color-singlet diquark condensates. At one loop, neither electric nor magnetic screening masses are generated; the electric sector is protected by the quark gaps, while the magnetic sector 
does not acquire the Meissner mass as the phase fluctuations of condensates do not couple to gauge fields. ${ }^{1}$ Including the gluon mass tempers the impact of medium polarization effects both in electric and magnetic propagators, while the presence of diquark gaps substantially weakens the electric corrections. These two effects seem necessary to reproduce the lattice and not to spoil the systematics of computations. As for corrections beyond the one loop, the case without diquark gaps has been studied in a Dyson-Schwinger framework, and this work shows that electric propagators are oversuppressed compared to the lattice data [55]. This suggests that the diquark gaps, among other nonperturbative effects, are indispensable to account for the lattice data.

Compared to the results based on pure perturbative gluons and quarks, the aforementioned quasiparticle picture substantially improves the consistency between the analytic results and the lattice's at zero temperature [11]. In this paper, we extend the analyses to the thermal medium.

One of new questions arising at a finite temperature is whether thermal excitations appear as quarks, or those excited quarks form color-singlet objects as in vacuum. For the schematic picture, see Fig. 1. Answering this question has the direct relevance to the quark-hadron continuity at a finite temperature [61] and to the quarkyonic matter conjecture which states that the bulk quark Fermi sea and baryonic structure are near the Fermi surface [62-74]. Also, since the lattice data available so far have not reached temperatures less than $T \sim 40 \mathrm{MeV}$, it is important to prepare analytic results whose setup is close to the lattice's.

The smallest temperature reached on the lattice is $\simeq 44 \mathrm{MeV}$. This temperature is not very low compared to the critical temperature of the diquark superfluidity, $T_{\mathrm{SF}} \simeq 90-120 \mathrm{MeV}$, found on the lattice. Therefore, we expect that the temperature corrections are not negligible. Below, in most cases, we will use the Bardeen-CooperSchrieffer (BCS) formulas, which are valid at weak coupling [75], as our baseline. In this approximation, the diquark gap at zero temperature is related to the critical temperature as ${ }^{2}$

$$
\Delta_{T=0} \simeq T_{\mathrm{SF}} / 0.57 \simeq 158-211 \mathrm{MeV},
$$

and the temperature dependence is

$$
\Delta_{T} \simeq \Delta_{T=0}\left(1-T / T_{\mathrm{SF}}\right)^{1 / 2} .
$$

\footnotetext{
${ }^{1}$ Similar situations have been discussed in two-flavor color superconductivity $[59,60]$.

${ }^{2}$ The critical temperature of the BCS is modified by the corrections such as the Popov and Gor'kov-Melik-barkhudarov corrections [76] even in the weak coupling limit, reducing the critical temperature by a factor $\simeq 2.2$. But these corrections are essentially modifications of effective interactions and reduce $\Delta$ in the same way. So the applicability of the BCS ratio $T_{c} \simeq 0.57 \Delta$ can be actually broader than that for the BCS estimate of each absolute value. See, e.g., Ref. [77] for a nice summary.
}

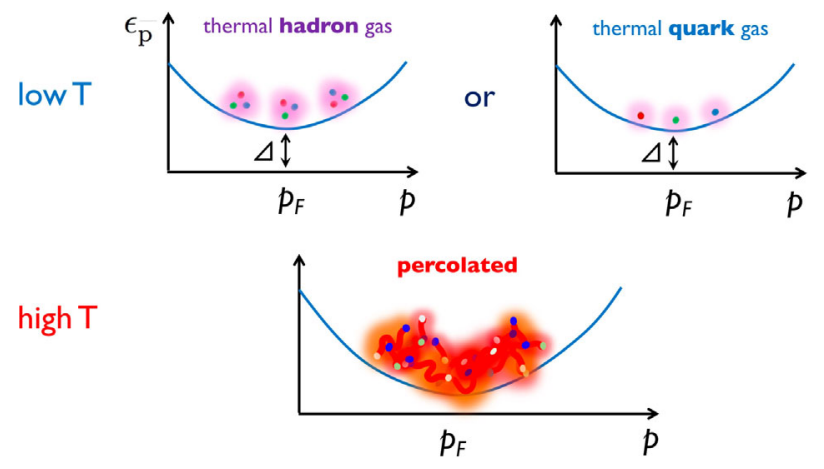

FIG. 1. Thermal excitations at low and high temperature. At low temperature, a thermal gas is made by hadrons or quarks. At high enough temperature, the gas becomes dense and get percolated. The percolation may happen within the superfluid phase if the phase space for low energy excitations is sufficiently large, e.g., at high density.

These should be reasonable estimates for a chemical potential $\mu_{q} \gtrsim 1 \mathrm{GeV}$ where we expect the validity of weak coupling pictures. We simply assume its extrapolation to a lower $\mu_{q}$ to give a useful guide.

Provided $\Delta_{T=0}=200 \mathrm{MeV}$, the gap at $T \simeq 44 \mathrm{MeV}$ is $\Delta_{T} \simeq 157 \mathrm{MeV}$. If thermal excitations are quarks, the Bolzmann factor is $\sim \mathrm{e}^{-\Delta_{T} / T} \sim \mathrm{e}^{-157 / 44} \sim 0.03$. But this suppression factor is not small enough to dominate over the phase space factor for low energy quarks, $\sim p_{F}^{2} \sqrt{\Delta_{T} T}$, where $p_{F}$ is the quark Fermi momentum. Thermal quarks behave as gapless particles as they are already excited and hence, contribute to the electric screening, in the same way as quarks in a normal phase. This introduces non-negligible effects in the electric sector. Meanwhile, in the magnetic sector, no magnetic mass is generated.

Another interesting possibility is that thermal excitations appear as hadrons, rather than individual thermal quarks. Then, at low temperature, thermal corrections from them are more strongly suppressed than in the thermal quark case. This should continue until thermal quarks and gluons are liberated through the overlap of thermally excited hadrons. This liberation of colors is driven by entropic effects that compensate the Boltzmann factor, like in the case of a Hagedorn gas [78-81]. In this picture of deconfinement, the critical temperature decreases as density increases, as more phase space is available for low energy excitations, and hence, the entropy increases (provided that $\Delta_{T}$ is not sensitive to density or $\mu_{q}$ ). With this picture in mind, we examine the temperature dependence of the lattice data at high density.

This paper is organized as follows: In Sec. II, we present the one-loop expressions for the polarization tensors from gluonic loops and quark loops. In Sec. III, after summarizing the setup used for the lattice simulations, we compare our one-loop results with the lattice's. Section IV is devoted to summary and discussions. 


\section{ANALYTIC RESULTS}

One-loop renormalized gluon polarization tensor in massive Yang-Mills (YM) theories has been computed in Ref. [20]. The results read

$$
\begin{aligned}
\Pi_{\mathrm{YM}}^{R}(k)= & \frac{g_{s}^{2} K^{2}}{192 \pi^{2}}\left\{111 s^{-1}-2 s^{-2}+\left(2-s^{2}\right) \ln (s)\right. \\
& +2\left(s^{-1}+1\right)^{3}\left(s^{2}-10 s+1\right) \ln (1+s) \\
& +\left(4 s^{-1}+1\right)^{3 / 2}\left(s^{2}-20 s+12\right) \\
& \left.\times \ln \left(\frac{\sqrt{4+s}-\sqrt{s}}{\sqrt{4+s}+\sqrt{s}}\right)-\left(s \leftrightarrow \mu_{R}^{2} / m_{g}^{2}\right)\right\},
\end{aligned}
$$

where $s=K^{2} / m_{q}^{2}$, with $K^{2}=k_{4}^{2}+\mathbf{k}^{2}$ being the squared four momenta in the Euclidean space, $m_{g}$ the gluon mass, and $g_{s}$ the coupling constant. The renormalization scale $\mu_{R}$ is chosen to be $\simeq 1 \mathrm{GeV}$, where the overall size of our gluon propagators is set to reproduce the lattice propagator at $\mu_{R}$. When we consider finite temperature gluon propagators, we neglect thermal gluon loops, which are suppressed at a temperature $(T \lesssim 200 \mathrm{MeV})$ substantially smaller than the energy of massive gluons $\left(m_{g} \sim 500 \mathrm{MeV}\right)$.

In a medium, the polarization effects caused by quarks are computed in the Nambu-Gor'kov formalism assuming the presence of a momentum independent gap $\Delta_{T}$. It is convenient to decompose the quark polarization tensor into the vacuum and medium pieces. Together with the vacuum counterterms, the renormalized polarization function is

$$
\Pi_{E, M}^{q, R}=\left.\Pi_{E, M}^{q}\right|_{\text {bare }}-k^{2} \delta_{Z_{g}}=\Pi_{\text {vac }}^{q, R}+\delta \Pi_{E, M}^{q},
$$

where the renormalized vacuum polarization and the medium corrections are

$$
\begin{aligned}
\Pi_{\text {vac }}^{q, R} & =\left.\Pi_{\text {vac }}^{q}\right|_{\text {bare }}-k^{2} \delta_{Z_{g}}, \\
\delta \Pi_{E, M}^{q} & =\left.\Pi_{E, M}^{q}\right|_{\text {bare }}-\left.\Pi_{\text {vac }}^{q}\right|_{\text {bare }} .
\end{aligned}
$$

The vacuum part is treated as in usual perturbation theories. The second term is the bare medium and vacuum contributions, both of which are UV divergent, and the subtraction leads to the UV finite expression. In the usual method to pick up poles of $p_{4}$ integrals, however, the implicit regularization of spatial momenta ${ }^{3}$ breaks the gauge invariance [58]. The artifacts automatically cancel if we use the same quark propagators for the medium and vacuum, but with such conditions, we would fail to capture the relevant physics associated with changes in quasiparticles. Fortunately, the Ward identity allows us to identify the gauge variant artifacts, so one can use gauge variant counterterms to cancel them. ${ }^{4}$ Including such procedure, the expression becomes [11]

$$
\delta \Pi_{E, M}^{q}=\delta_{\Delta S} \Pi_{E, M}^{q}+\delta_{\Delta \mu_{q}} \Pi_{E, M}^{q} .
$$

The first term takes care of changes in quark propagators and is computed in the dimensional regularization,

$$
\begin{aligned}
\left.\delta_{\Delta S} \Pi(k)\right|_{\text {dim reg }}= & -K^{2} \frac{g_{s}^{2}}{2 \pi^{2}} \\
& \times \int_{0}^{1} d x x(1-x) \ln \frac{\left(\tilde{M}_{q}\right)^{2}+x(1-x) K^{2}}{\left(M_{q}^{\text {vac }}\right)^{2}+x(1-x) K^{2}},
\end{aligned}
$$

with $\tilde{M}_{q}=\sqrt{\Delta_{T}^{2}+M_{q}^{2}}$. Here, $M_{q}^{\text {vac }}$ is the constituent quark mass in vacuum whose value is set to $M_{q}^{\mathrm{vac}}=0.3 \mathrm{GeV}$, while $M_{q}$ is the mass at high density, which should be close to the current quark mass. We set $M_{q}=0.1 \mathrm{GeV}$, considering the large pion mass, $m_{\pi} \sim 0.7 \mathrm{GeV}$. The form of $\tilde{M}_{q}$ is uniquely fixed to cancel the artifacts in the second term in Eq. (6). The expression is given by $\left(\int_{\mathbf{q}} \equiv \int \mathrm{d}^{3} \mathbf{q} /(2 \pi)^{3}\right)$,

$$
\begin{aligned}
\left.\delta_{\Delta \mu_{q}} \Pi_{E, M}^{q}(k)\right|_{3 \mathrm{~d} \text { reg }}= & \left.\Pi_{E, M}^{q}(k)\right|_{\text {bare }} \\
& -\left.\Pi_{E, M}^{q}(k)\right|_{\text {bare }} ^{M_{q} \rightarrow \tilde{M}_{q} ; \mu_{q} \rightarrow 0 ; \Delta \rightarrow 0},
\end{aligned}
$$

where

$$
\left.\Pi_{E, M}^{q}(k)\right|_{\text {bare }}=g_{s}^{2} \sum_{s, s^{\prime}=\mathrm{p}, \mathrm{a}} \int_{\mathbf{q}} \mathcal{K}_{E, M}^{s s^{\prime}}\left(\mathbf{q}_{+}, \mathbf{q}_{-}\right)\left[\mathcal{C}_{E, M}^{s s^{\prime}}\left(\mathbf{q}_{+}, \mathbf{q}_{-}\right) \mathcal{G}_{s s^{\prime}}\left(q_{+}, q_{-}\right)+\tilde{\mathcal{C}}_{E, M}^{s s^{\prime}}\left(\mathbf{q}_{+}, \mathbf{q}_{-}\right) \tilde{\mathcal{G}}_{s s^{\prime}}\left(q_{+}, q_{-}\right)\right]
$$

Here, $\mathbf{q}_{ \pm}=\mathbf{q} \pm \mathbf{k} / 2$. Below, we write $E_{q_{ \pm}}=\sqrt{M_{q}^{2}+\mathbf{q}_{ \pm}^{2}}$ and the excitation energies for quasiparticles and quasiantiparticles as $\epsilon_{\mathrm{p}}^{ \pm}=\sqrt{\left(E_{q_{ \pm}}-\mu_{q}\right)^{2}+\Delta_{T}^{2}}$ and $\epsilon_{\mathrm{a}}^{ \pm}=\sqrt{\left(E_{q_{ \pm}}+\mu_{q}\right)^{2}+\Delta_{T}^{2}}$, respectively.

\footnotetext{
${ }^{3}$ We take $|\mathbf{p}| \rightarrow \infty$ only after picking up the residue.

${ }^{4}$ The procedure here uniquely specifies the gauge variant counterterms for a given external momentum $k$. But, in principle, there are still possibilities that the dependence on the gauge variant regularization would enter gauge invariant quantities as finite terms. There are, at least to us, no obvious way to identify such contributions. We leave this problem for the future.
} 
The explicit forms of these factors are as follows: the kinematic factors are

$$
\begin{aligned}
& \mathcal{K}_{E}^{\mathrm{pp}}=\mathcal{K}_{E}^{\mathrm{aa}}=1+\frac{\vec{q}^{2}-\mathbf{k}^{2} / 4+M_{q}^{2}}{E_{q_{+}} E_{q_{-}}}, \\
& \mathcal{K}_{E}^{\mathrm{pa}}=\mathcal{K}_{E}^{\mathrm{ap}}=1-\frac{\vec{q}^{2}-\mathbf{k}^{2} / 4+M_{q}^{2}}{E_{q_{+}} E_{q_{-}}}, \\
& \mathcal{K}_{M}^{\mathrm{pp}}=\mathcal{K}_{M}^{\mathrm{aa}}=-1+\frac{(|\vec{q}| \cos \theta)^{2}-\mathbf{k}^{2} / 4+M_{q}^{2}}{E_{q_{+}} E_{q_{-}}}, \\
& \mathcal{K}_{M}^{\mathrm{pa}}=\mathcal{K}_{M}^{\mathrm{ap}}=-1-\frac{(|\vec{q}| \cos \theta)^{2}-\mathbf{k}^{2} / 4+M_{q}^{2}}{E_{q_{+}} E_{q_{-}}},
\end{aligned}
$$

where $\hat{k}=\mathbf{k} /|\mathbf{k}|$ and $\cos \theta$ is the angle between $\mathbf{q}$ and $\mathbf{k}$; the coherence factors are

$$
\begin{aligned}
\mathcal{C}_{E, M}^{\mathrm{pp}} & =\frac{1}{2}\left(1-\frac{\left(E_{q_{+}}-\mu_{q}\right)\left(E_{q_{-}}-\mu_{q}\right) \pm\left|\Delta_{T}\right|^{2}}{\epsilon_{\mathrm{p}}^{+} \epsilon_{\mathrm{p}}^{-}}\right), \\
\mathcal{C}_{E, M}^{\mathrm{aa}} & =\frac{1}{2}\left(1-\frac{\left(E_{q_{+}}+\mu_{q}\right)\left(E_{q_{-}}+\mu_{q}\right) \pm\left|\Delta_{T}\right|^{2}}{\epsilon_{\mathrm{a}}^{+} \epsilon_{\mathrm{a}}^{-}}\right), \\
\mathcal{C}_{E, M}^{\mathrm{pa}} & =\frac{1}{2}\left(1+\frac{\left(E_{q_{+}}-\mu_{q}\right)\left(E_{q_{-}}+\mu_{q}\right) \mp\left|\Delta_{T}\right|^{2}}{\epsilon_{\mathrm{p}}^{+} \epsilon_{\mathrm{a}}^{-}}\right),
\end{aligned}
$$

and

$$
\begin{aligned}
& \tilde{\mathcal{C}}_{E, M}^{\mathrm{pp}}=\frac{1}{2}\left(1+\frac{\left(E_{q_{+}}-\mu_{q}\right)\left(E_{q_{-}}-\mu_{q}\right) \pm\left|\Delta_{T}\right|^{2}}{\epsilon_{\mathrm{p}}^{+} \epsilon_{\mathrm{p}}^{-}}\right), \\
& \tilde{\mathcal{C}}_{E, M}^{\mathrm{aa}}=\frac{1}{2}\left(1+\frac{\left(E_{q_{+}}+\mu_{q}\right)\left(E_{q_{-}}+\mu_{q}\right) \pm\left|\Delta_{T}\right|^{2}}{\epsilon_{\mathrm{a}}^{+} \epsilon_{\mathrm{a}}^{-}}\right), \\
& \tilde{\mathcal{C}}_{E, M}^{\mathrm{pa}}=\frac{1}{2}\left(1-\frac{\left(E_{q_{+}}-\mu_{q}\right)\left(E_{q_{-}}+\mu_{q}\right) \mp\left|\Delta_{T}\right|^{2}}{\epsilon_{\mathrm{p}}^{+} \epsilon_{\mathrm{a}}^{-}}\right),
\end{aligned}
$$

where $\mathcal{C}_{E, M}^{\mathrm{pa}}\left(q_{+}, q_{-}\right)=\mathcal{C}_{E, M}^{\mathrm{ap}}\left(q_{-}, q_{+}\right)$and $\tilde{\mathcal{C}}_{E, M}^{\mathrm{pa}}\left(q_{+}, q_{-}\right)=$ $\tilde{\mathcal{C}}_{E, M}^{\mathrm{ap}}\left(q_{-}, q_{+}\right)$; finally, the propagator part is

$$
\begin{aligned}
\mathcal{G}_{s s^{\prime}}\left(q_{+}, q_{-}\right)= & \frac{1}{2}\left(1-n\left(\epsilon_{s}^{+}\right)-n\left(\epsilon_{s^{\prime}}^{-}\right)\right) \\
& \times\left(\frac{1}{\mathrm{i} k_{4}+\epsilon_{s}^{+}+\epsilon_{s^{\prime}}^{-}}+\frac{1}{-\mathrm{i} k_{4}+\epsilon_{s}^{+}+\epsilon_{s^{\prime}}^{-}}\right),
\end{aligned}
$$

and

$$
\begin{aligned}
\tilde{\mathcal{G}}_{s s^{\prime}}\left(q_{+}, q_{-}\right)= & -\frac{1}{2}\left(n\left(\epsilon_{s}^{+}\right)-n\left(\epsilon_{s^{\prime}}^{-}\right)\right) \\
& \times\left(\frac{1}{\mathrm{i} k_{4}+\epsilon_{s}^{+}-\epsilon_{s^{\prime}}^{-}}+\frac{1}{-\mathrm{i} k_{4}+\epsilon_{s}^{+}-\epsilon_{s^{\prime}}^{-}}\right) .
\end{aligned}
$$

The function $n(x)$ is the Fermi-Dirac distribution $n(x)=$ $1 /\left(\mathrm{e}^{\beta(x-\mu)}+1\right)$. Below, we focus on the static behaviors of the gluon propagators at $k_{4}=0$, where the results are most sensitive to the nonperturbative effects. Table I summarizes the coherence and kinematical factors for electric and magnetic gluons, and the factors from the propagators.

The zero temperature limit was discussed in the previous papers [11,58]; the electric part is dominated by the particle-hole contributions for any phases, as one can see from the kinematic factor. Then, in the case of color-singlet diquark condensed phases, the coherence factor vanishes due to the quark gap, while the gaps also introduce the infrared cutoff ${ }^{5} \sim \Delta_{T}$ in the propagator factor $\mathcal{G}_{\mathrm{pp}}$. These facts together lead to vanishing electric polarization for $\mathbf{k} \rightarrow 0$. On the other hand, the magnetic contributions come from everywhere, from (pp), (pa), and (aa), which are correlated through the gauge invariance; for quark propagators in normal or color-singlet diquark condensed phases, all the contributions are assembled to cancel, leaving the vanishing magnetic contributions.

At a finite temperature, the magnetic sector for the static limit is unchanged, while the electric sector is no longer protected by the quark gap. There are thermally excited quarks that can be easily perturbed by external fields; these thermal quarks are gapless. Moreover, they are not arranged into color singlet objects. Accordingly, the static limit $\left(k_{0}=0,|\mathbf{k}| \rightarrow 0\right)$ yields

$$
\begin{aligned}
& \tilde{\mathcal{G}}_{\mathrm{pp}} \rightarrow-\frac{\partial n\left(\epsilon_{\mathrm{p}}\right)}{\partial \epsilon_{\mathrm{p}}}=\frac{1}{T} \frac{\mathrm{e}^{\epsilon_{\mathrm{p}} / T}}{\left(\mathrm{e}^{\epsilon_{\mathrm{p}} / T}+1\right)^{2}} \sim \frac{\mathrm{e}^{-\epsilon_{\mathrm{p}} / T}}{T}, \\
& \tilde{\mathcal{C}}_{E}^{\mathrm{pp}} \rightarrow 1 .
\end{aligned}
$$

Here, the coherence factor is 1 , like in a normal phase. This contribution adds the Debye mass to the electric sector. This correction is very sensitive to the size of gap that controls the abundance of thermal quarks. At a low temperature, its size is $\sim \mathrm{e}^{-\Delta_{T} / T} / T$, exponentially suppressed. As the temperature approaches the critical temperature of diquark condensations, $\Delta_{T}$ approaches zero; here, the factor $\tilde{\mathcal{G}}_{\mathrm{pp}}$ is no longer exponentially suppressed and becomes $\sim 1 / T$, and combining it with the phase space factor $\sim T p_{F}^{2}$ and coupling constants leads to the Debye mass of $m_{D}^{2} \sim g_{s}^{2} T^{-1}\left(T p_{F}^{2}\right) \sim g_{s}^{2} p_{F}^{2}$.

\section{COMPARISON WITH THE LATTICE DATA}

As in our previous study [11], we will use the data in Ref. [28] based on the gauge configurations of unimproved Wilson gauge action with two flavors of unimproved Wilson quarks. The pion mass is $m_{\pi}=717(25) \mathrm{MeV}$, so the onset chemical potential of the baryon density is

\footnotetext{
${ }^{5}$ If $\Delta_{T}=0$, the $\mathcal{C}_{\mathrm{pp}} \mathcal{G}_{\mathrm{pp}}$ becomes finite in the static limit, producing the Debye mass.
} 
$\mu_{c}=m_{\pi} / 2 \simeq 358 \mathrm{MeV} .^{6}$ As the explicit chiral symmetry breaking is so large, we regard pions as two constituent quarks in the same way as $\rho$ mesons or other mesons. The critical temperature of deconfinement, $T_{D}$, is defined by the Polyakov loop and $T_{D}=217(23) \mathrm{MeV}$.

Our previous paper has compared the one-loop results with the lattice data for $\beta=1.9$ and $N_{t} \times N_{s}^{3}=24 \times 16^{3}$ with the inverse lattice spacing $a^{-1} \simeq 1.06 \mathrm{GeV}$, the spatial size $L_{s} \simeq 2.98 \mathrm{fm}$, and the temporal size $L_{t} \simeq 4.46 \mathrm{fm}$. While the data for this $L_{t}$ was presented as the zero temperature result [28], this corresponds to $T \simeq 44 \mathrm{MeV}$ if we interpret $1 / L_{t}$ as the temperature. At zero density, the result at $L_{t}>L_{s}$ is often interpreted as the zero temperature result as the finite volume makes the lowest momentum $\sim 1 / L_{s}$ and hence, lifts up the energy. But at finite density, there is the Fermi sea, and large momentum states do not necessarily mean high energy states. ${ }^{7}$ Following Ref. [27], we literally take $1 / L_{t}$ as the temperature. These temperature corrections were not taken into account in our previous analyses, and we shall include these corrections.

In this paper, we compare our finite temperature expression with the lattice data. The data set for $\beta=2.1$ and $N_{s}=16$ with the inverse lattice spacing $a^{-1}=1.41 \mathrm{GeV}$ covers the wide range of temperatures, and this data set will be used in this work. The spatial size is $L_{s} \simeq 2.21 \mathrm{fm}$, and the temporal size covers the temperature range from $T \simeq$ $44 \mathrm{MeV} \quad\left(N_{t}=32\right)$ to $\simeq 353 \mathrm{MeV} \quad\left(N_{t}=4\right)$. See Appendix B of Ref. [28] where details of the simulation setup are summarized.

In order to describe the finite temperature, it is crucial to know the size and temperature dependence of the gap. As a baseline, we simply assume the BCS expressions in Eqs. (1) and (2). The critical temperature for the diquark condensation, $T_{\mathrm{SF}}$ is $\simeq 90-120 \mathrm{MeV}$ for $\mu_{q}=705 \mathrm{MeV}$, and at high density, it is not very sensitive to changes in $\mu_{q}$. Thus, throughout our analyses, we take

$$
\Delta_{T=0}=200 \mathrm{MeV},
$$

for the zero temperature gap, and the corresponding critical temperature is

$$
T_{\mathrm{SF}}=114 \mathrm{MeV} \text {. }
$$

Below, we will substitute these expressions unless otherwise stated.

For comparisons of our results with the lattice data, we need to multiply an overall factor as the latter results were not renormalized. We use the expression,

$$
D_{\mathrm{E}, \mathrm{M}}^{\text {lattice }}(k)=Z_{\text {lattice }} D_{E, M}^{\text {analytic }}(k),
$$

\footnotetext{
${ }^{6}$ The impact of explicit symmetry breaking was examined in detail by chiral effective models; see Ref. [54].

${ }^{7}$ We acknowledge Professors A. Maas and J. Skullerud for instructions on this point.
}

and chose $Z_{\text {lattice }}=3.0$ throughout. We use the lattice results in Ref. [28], the bottom panels in Fig. 11, top panels in Fig. 16, and top panels in Fig. 19. The domains we study in Sec.III A-C are summarized in Fig. 2, together with a schematic phase diagram.

\section{A. The $\mu_{q}$ dependence at $T \simeq 44 \mathrm{MeV}$}

We first consider the low temperature case. For this case, the dependence of gluon propagators on $m_{g}, \Delta_{T=0}, \alpha_{s}$, and momenta were studied in detail in Ref. [11], so in this paper, we limit ourselves to fewer cases.

To get ideas about the overall impact of parameters and the tendency of electric and magnetic propagators, in Fig. 3, we first show the $m_{g}$ and $\Delta_{T=0}$ dependence of gluon propagators (see also Ref. [11]). The $\alpha_{s}$ is fixed to 1.0. We note that there is disparity in the magnitudes of electric and magnetic propagators already in vacuum due to the fact that the lattice setup is anisotropic in temporal and spatial directions. Keeping this in mind, we vary $m_{g}$ by $\pm 10 \%$, which changes the magnitudes of propagators by $\sim 10 \%$ in the IR limit. The $\Delta_{T=0}$ dependence is very large in the electric sector, but in the magnetic sector, such a dependence is not visible. In a medium, the analytic results show that the magnetic propagators have the paramagnetic enhancement at finite momenta compared to the vacuum case. It is difficult to see this enhancement in the lattice results perhaps because the second smallest momentum is too large, $|\mathbf{k}| \simeq 0.55 \mathrm{GeV}$.

Below, we examine the $\mu_{q}$ dependence of static gluon propagators at three momentum data points. Shown in Fig. 4 is the $\mu_{q}$ dependence of the electric (upper) and magnetic (lower) gluon propagators $D_{E, M}(k)$ at momenta $|\mathbf{k}|=0.0,0.55,1.08 \mathrm{GeV}$. The lattice data are shown with error bars, and the corresponding analytic results are shown with three lines; from bold to thin lines, we show the results for $\alpha_{s}=0.0,0.5,1.0$. For the electric and magnetic masses (tree level), we chose $m_{E}=0.6 \mathrm{GeV}$ and $m_{M}=0.5 \mathrm{GeV}$, respectively, to get good overall fit.

The overall behaviors are that, in both sectors, the gluon propagators are largely insensitive to $\mu_{q}$, except $\mu_{q} \gtrsim 0.8 \mathrm{GeV}$, where the propagator at $|\mathbf{k}|=0$ starts to get

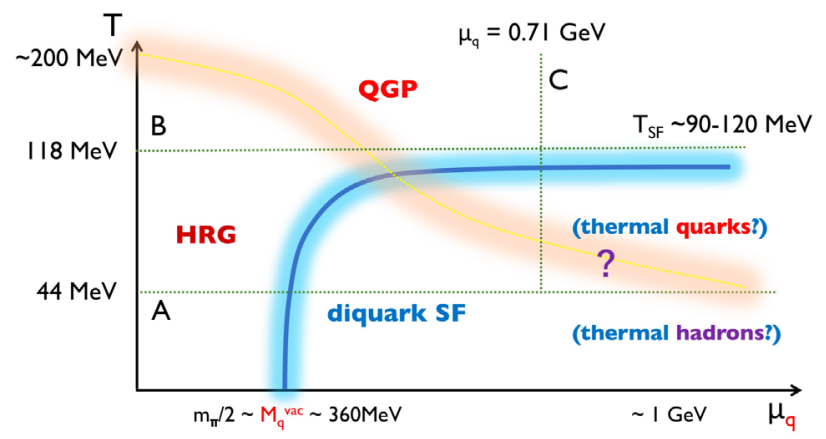

FIG. 2. A schematic phase diagram. The lines A, B, C indicate the domains analyzed in Secs. IIIA-IIIC. See also Fig. 10 in Ref. [30] and Fig. 2 in Ref. [46] based on the Polyakov loop. 

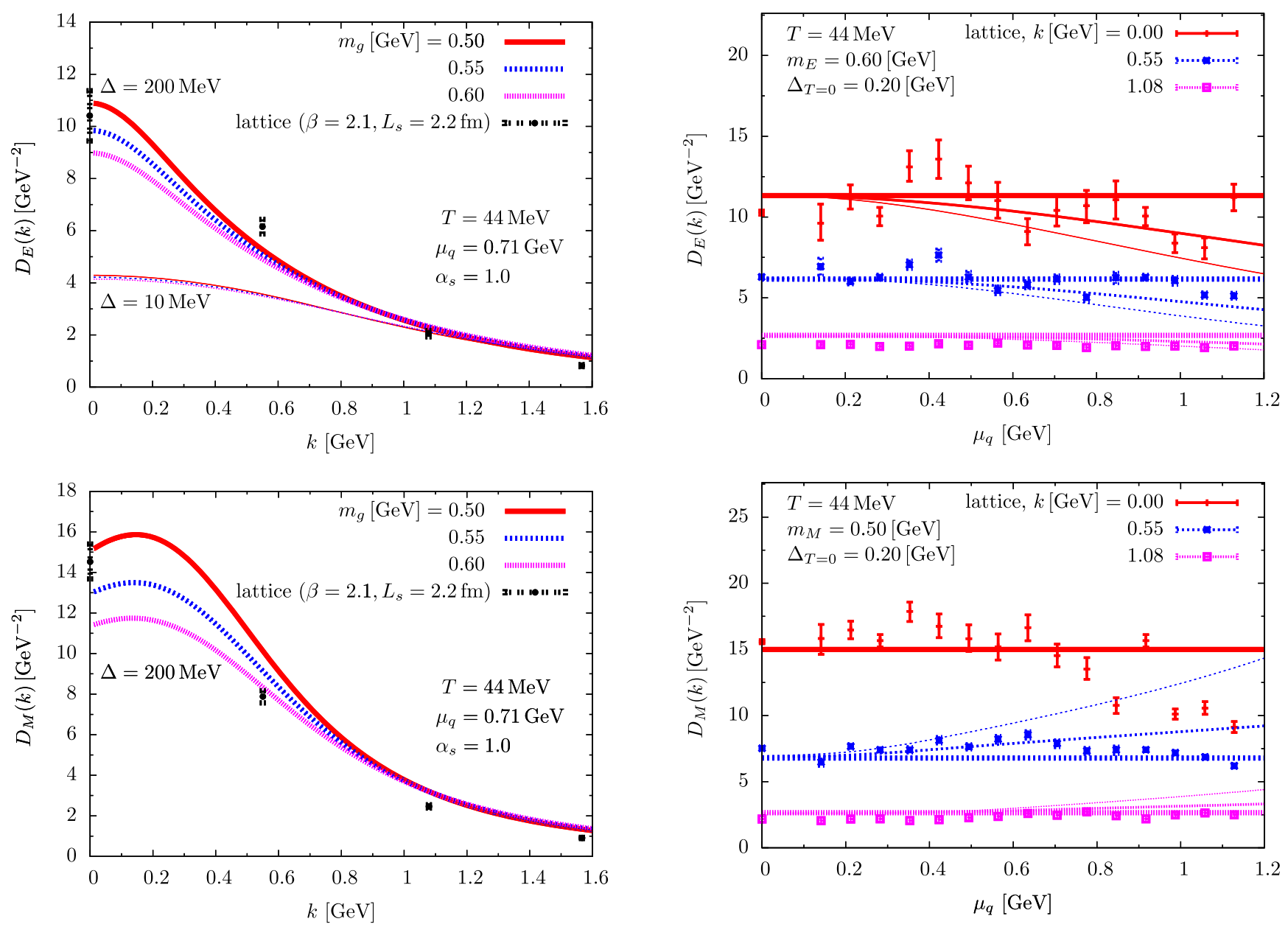

FIG. 3. The static propagators at $\mu_{q}=0.71 \mathrm{GeV}$ and $T \simeq 44 \mathrm{MeV}$, for $\alpha_{s}=1.0$. The upper (lower) panel is for electric (magnetic) propagators. For the electric propagators, we compare the $\Delta_{T=0}=200 \mathrm{MeV}$ and $10 \mathrm{MeV}$, while in the magnetic sector the dependence on $\Delta_{T}$ is not visible for the range 10-200 MeV.

suppressed gradually. It is not easy which $\alpha_{s}=0.0$ or 0.5 should be regarded as the better choice, but it seems that the choice $\alpha_{s}=1.0$ leads to too large changes, especially at momenta $|\mathbf{k}|=0.55 \mathrm{GeV}$, in the electric sector due to the thermal Debye screening and in the magnetic sector due to the paramagnetic enhancement.

Here, we mention briefly the trend seen in the magnetic sector for $\mu_{q} \gtrsim 0.8 \mathrm{GeV}$ where the magnetic propagators seem to decrease for increasing $\mu_{q}$. This trend cannot be explained in our one-loop calculations. We note that in this domain of $\mu_{q}$ and $T$, the Polyakov loop starts to grow [30]. So we guess that the trend for $\mu_{q} \gtrsim 0.8 \mathrm{GeV}$ in Fig. 4 originates from the beyond one-loop effects; presumably, we need to address the modification of gluon mass at the nonperturbative level.

\section{B. The $\mu_{q}$ dependence at $T \simeq 118 \mathrm{MeV}$}

Next we turn into higher temperature, $T \simeq 118 \mathrm{MeV}$, and examine the $\mu_{q}$ dependence of the gluon propagators

FIG. 4. The $\mu_{q}$ dependence of the gluon propagators at spatial momenta $|\mathbf{k}|=0.0,0.55,1.08 \mathrm{GeV}$ and at a temperature $T \simeq 44 \mathrm{MeV}$. The upper (lower) panel is for electric (magnetic) propagators. The plots with error bars correspond to the lattice data. From bold to thin lines, they correspond to the analytic results at $\alpha_{s}=0.0,0.5,1.0$, and the $\Delta_{T=0}=0.2 \mathrm{GeV}$ and $\Delta_{T=44} \simeq 157 \mathrm{MeV}$.

(Fig. 5). We reduce the gluon mass slightly, $\sim 10 \%$, instead of manifestly computing the gluon loop at a finite temperature. At this temperature, the diquark condensates melt or are vanishingly small. Also, the lattice results show that the Polyakov loop is large, meaning the abundance of thermal quarks. Therefore, we may expect that the lattice data can be explained by computations based on deconfined thermal quarks.

The comparison between analytic results and the lattice results supports this picture. The electric propagators are drastically reduced by the Debye screening as $\mu_{q}$ increases, and the trend in the lattice data are reproduced for all spatial momenta. As for the magnetic sector, we have not fully understood the growth of the propagator at $|\mathbf{k}|=0$ for increasing $\mu_{q}$, but the growing behavior at $|\mathbf{k}|=0.55$ and $1.08 \mathrm{GeV}$ is reasonably consistent with the paramagnetic enhancement in analytic computations. 

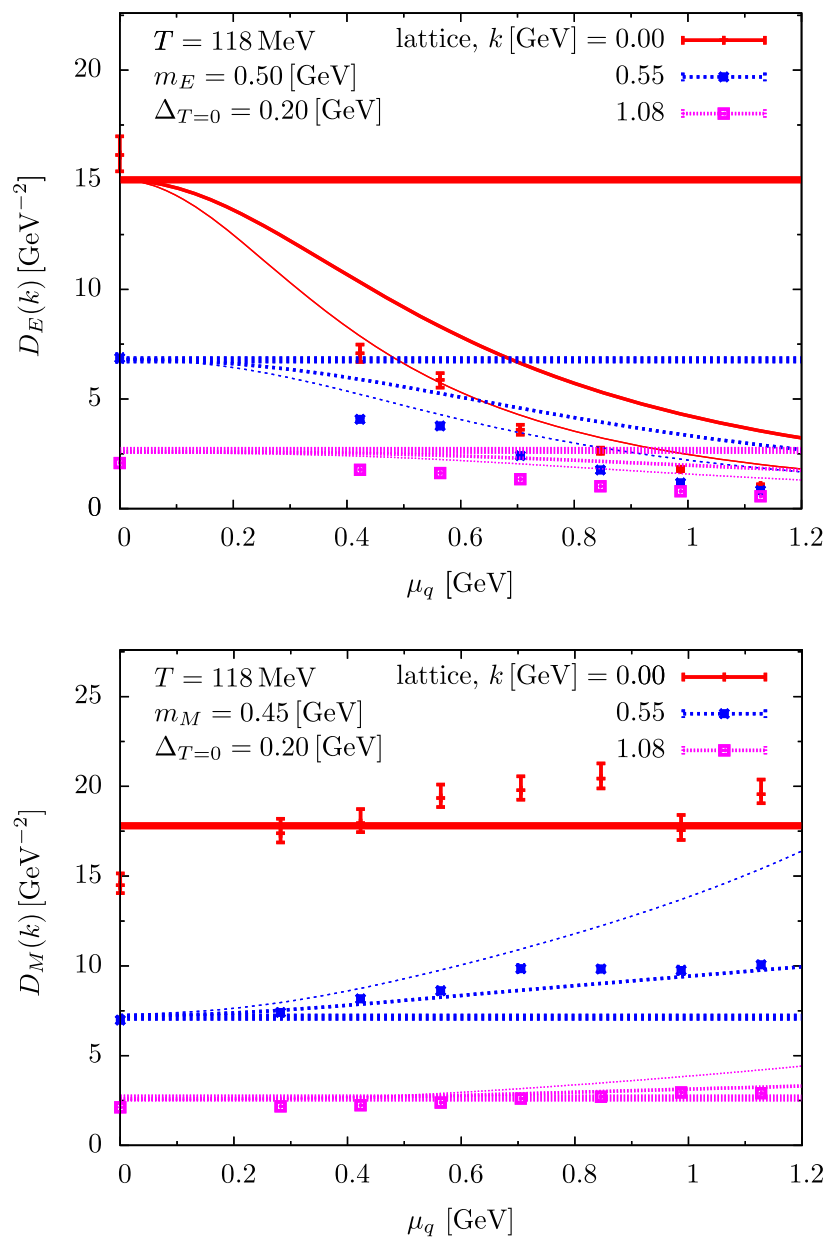

FIG. 5. The same as Fig. 4 except that the temperature is $T \simeq 118 \mathrm{MeV}$, and $m_{E}$ and $m_{M}$ change slightly. The temperature is higher than $T_{\mathrm{SF}}$, so $\Delta_{T=118}=0$.

As for the $\alpha_{s}$ dependence, although we have not tried a fine-tuning, the results for the range of $\alpha_{s}=0.5-1.0$ seem to give reasonable descriptions for the $\mu_{q}$ dependence.

\section{The $\boldsymbol{T}$ dependence at $\boldsymbol{\mu}_{q}=0.71 \mathrm{GeV}$}

Finally, we fix $\mu_{q}=0.71 \mathrm{GeV}$ and examine the temperature dependence of gluon propagators. The lowest temperature is $T \simeq 44 \mathrm{MeV}$, and the next is $\simeq 108 \mathrm{MeV}$, which is beyond or close to $T_{\mathrm{SF}}$. As we have not included the temperature corrections to gluon loops, we stop the comparison around $\simeq 150-200 \mathrm{MeV}$, where thermal gluons of densities of $\sim T^{3}$ should not be ignored.

For the electric sector, the most notable is the behavior of the softest mode, $D_{E}(|\mathbf{k}|=0)$. In analytic results assuming thermal quark excitations, the damping starts to take place around $T \simeq 30 \mathrm{MeV}$ and is completed around $T=T_{\mathrm{SF}}$ reaching $\sim\left(g_{s} p_{F}\right)^{-2}$, as expected from usual Debye screening arguments. Meanwhile, the lattice data seem to show slower damping. To adjust the analytic results to the lattice data, we would need gaps about $\Delta_{T=0} \sim 300-400 \mathrm{MeV}$, which are perhaps too large. A more reasonable way to reconcile the analytic results with the lattice data is to modify the temperature dependence of $\Delta_{T}$; if the $\Delta_{T}$ is stiffer against thermal corrections, i.e., if the abundance thermal quarks are suppressed, then $D_{E}(|\mathbf{k}|=0)$ is more stable at low temperature and more quickly melt near $T_{\mathrm{SF}}$ than in the present result.

These considerations bring us to the conjecture that thermal excitations are color-singlet hadrons. If true, thermal loops coupled to external gluon lines are those of hadrons, rather than of quarks. Accordingly, the availability of thermal particles are controlled by the Boltzmann factor and is $\sim \mathrm{e}^{-2 \Delta_{T} / T}$, rather than $\sim \mathrm{e}^{-\Delta_{T} / T}$, because hadrons contain at least two excited quarks out of condensates. At the beginning, hadrons form a dilute gas, and with increasing $T$, those hadrons overlap, allowing thermal quark descriptions. We expect this starts to take place at $T<T_{\mathrm{SF}}$ gradually. As for the magnetic sector, the softest mode, $D_{M}(|\mathbf{k}|=0)$, shows slight enhancement around $T \simeq T_{\mathrm{SF}}$, but the overall behavior is rather insensitive to $T$.

\section{SUMMARY AND DISCUSSION}

We have examined the Landau gauge gluon propagators at a finite density and temperature. With analytic oneloop expressions added to massive vacuum gluon propagators, we compare the analytic results with the lattice data in the domains (i) $T \simeq 44 \mathrm{MeV}$ for $\mu_{q}=0-1.2 \mathrm{GeV}$, (ii) $T \simeq 118 \mathrm{MeV}$ for $\mu_{q}=0-1.2 \mathrm{GeV}$, and (iii) $T \simeq$ 0-200 MeV for $\mu_{q}=0.71 \mathrm{GeV}$, for which the lattice data are available for several spatial momenta. The gap and critical temperature of the diquark condensation is assumed to be $\Delta_{T=0}=200 \mathrm{MeV}$ and $T_{\mathrm{SF}} \simeq 114 \mathrm{MeV}$, respectively.

The behaviors beyond $T_{\mathrm{SF}}$ are overall consistent with the standard descriptions based on the thermal quark loops. The electric sector acquires the Debye mass $\sim g_{s} p_{F}$ from gapless modes. In the magnetic sector, the paramagnetic behaviors at finite momenta are in agreement between the analytic one-loop results and the lattice's. But the analytic results do not describe the slight enhancement in the static mode of the lattice results.

At a low temperature $T \simeq 44 \mathrm{MeV}$, the lattice data show that the electric and magnetic propagators show only rather weak dependence on the medium effects. To reproduce the behavior in the electric sector, it is essential to include the diquark gap; otherwise, the Debye screening strongly changes the analytic results. In the previous study, we found that $\Delta_{T=0} \simeq 200 \mathrm{MeV}$ gives the results consistent with the lattice's, while the choice $\Delta_{T=0} \simeq 100 \mathrm{MeV}$ is a bit too small to reproduce the data at finite momenta. In contrast, the magnetic sector in the analytic results remains insensitive to details of $\Delta_{T}$.

There are two significant features in the lattice results that cannot be understood in simple terms. The first is the magnetic propagators in the IR limit; at a low temperature, the magnetic propagators seem slightly weakened as 

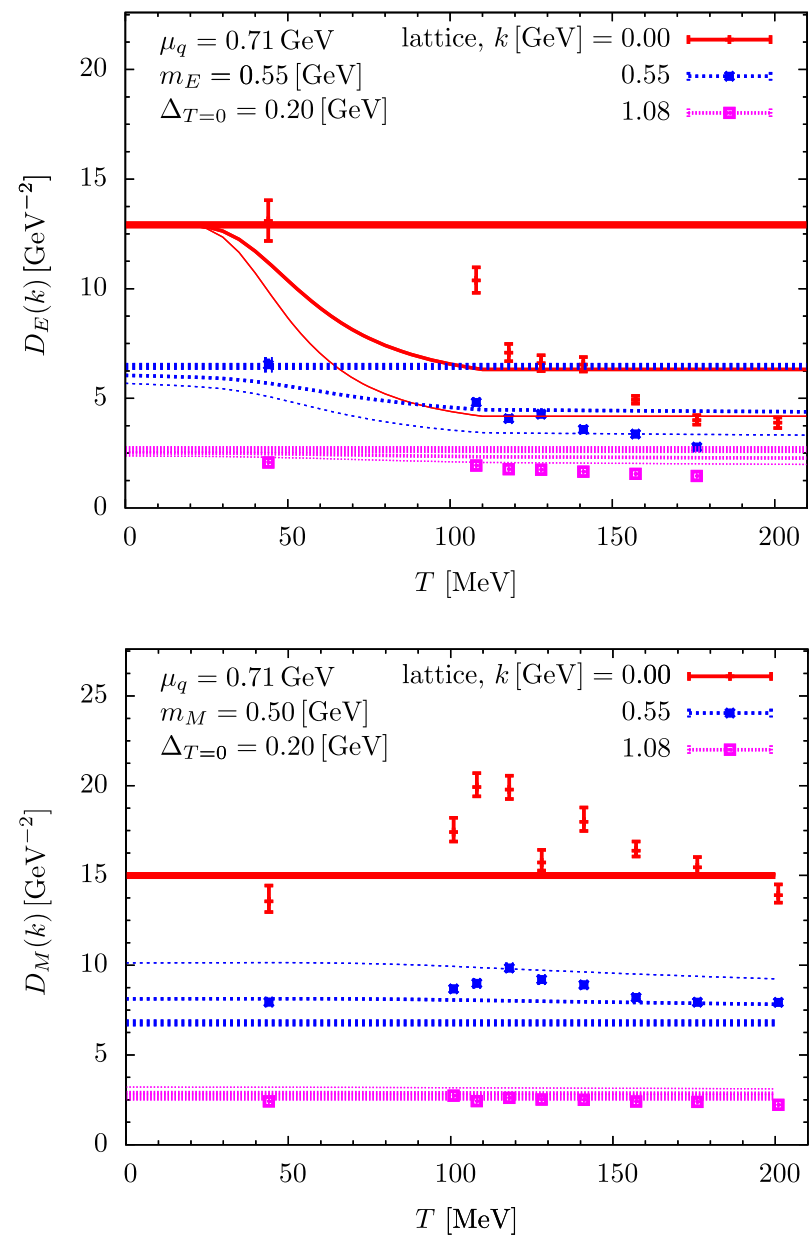

FIG. 6. The $T$ dependence of the gluon propagators at spatial momenta $|\mathbf{k}|=0.0,0.55,1.08 \mathrm{GeV}$ and at $\mu_{q} \simeq 0.71 \mathrm{GeV}$. The upper (lower) panel is for electric (magnetic) propagators. The plots with error bars correspond to the lattice data. From bold to thin lines, they correspond to the analytic results at $\alpha_{s}=0.0,0.5$, 1.0 , and the $\Delta_{T=0}=0.2 \mathrm{GeV}$.

density increases, while at a high temperature, they slightly get enhanced. One solution would be the finite volume artifacts whose impacts should be strongest for soft modes. If not, we need treatments beyond one-loop and/or nonperturbative framework.

The second is the low temperature dependence of the electric propagator at a high density, $\mu_{q} \simeq 0.71 \mathrm{GeV}$. In our calculations, thermal quarks are gapless and contribute to the Debye screening already at $T \simeq 44 \mathrm{MeV}$, and the screening is very strong at $T \simeq 108 \mathrm{MeV}$ (the second lowest temperature in Fig. 6). The electric screening at $T \simeq$ $108 \mathrm{MeV}$ seems substantially stronger than the lattice data. Again, the discrepancy can be finite volume artifacts, but in that case, the lattice result must be reduced considerably, by $\sim 30 \%-40 \%$. If this discrepancy remains true for simulations at a bigger volume, it will raise questions on the nature of thermal excitations in a high density matter, i.e., whether thermal excitations below $T_{\mathrm{SF}}$ are thermal quarks or thermal hadrons in superfluid quark matter.
TABLE I. The coherence and kinematical factors for electric and magnetic gluons, and the factors from the propagators at $|\mathbf{q}|=p_{F}$, where $p_{F}$ is the quark Fermi momentum such that $E\left(p_{F}\right)=\mu_{q}$.

\begin{tabular}{cccccc}
\hline \hline & $\mathcal{C}_{E}$ & $\mathcal{K}_{E}$ & $\mathcal{C}_{M}$ & $\mathcal{K}_{M}$ & $\mathcal{G}\left(|\mathbf{q}|=p_{F}\right)$ \\
\hline pp & $\sim \mathbf{q} \cdot \mathbf{k}$ & 2 & $\left(\frac{\Delta_{T}}{\epsilon_{\mathrm{p}}(q)}\right)^{2}$ & $\left(\frac{\mathbf{q}}{E_{q}} \sin \theta\right)^{2}$ & $\sim \frac{1}{\mathbf{q} \cdot \mathbf{k}+\Delta_{T}^{2}}$ \\
aa & $\sim \mathbf{q} \cdot \mathbf{k}$ & 2 & $\left(\frac{\Delta_{T}}{\epsilon_{\mathrm{a}}(q)}\right)^{2}$ & $\left(\frac{\mathbf{q}}{E_{q}} \sin \theta\right)^{2}$ & $\sim \frac{1}{p_{F}}$ \\
pa & finite & $\sim \mathbf{k}^{2}$ & Finite & -2 & $\sim \frac{1}{p_{F}}$ \\
\hline \hline
\end{tabular}

The consequence of thermal excitations are reflected in quantities such as entropies and Polyakov loops. At a low temperature, the entropy of a hadron gas is much smaller than of a thermal quark gas,

$$
s_{\mathrm{H}} / s_{\mathrm{Q}} \sim \mathrm{e}^{-\Delta_{T} / T}
$$

as hadronic excitations must contain more than one quark and its energy cost is at least $\sim \Delta_{T}$. This low temperature regime continues, as in a Hagedorn gas, until the Boltzmann suppression is compensated by entropic effects, i.e., drastic growth in the number of hadronic excitations and subsequent overlap of hadrons. This Hagedorn type description can give a reasonable description of colordeconfinement at large density, in the same spirit as the transition from a hadron resonance gas to a quark-gluonplasma at $\mu_{q}=0$. Below such a temperature, the matter can be regarded as a quarkyonic matter.

As the entropy is the main ingredient here, the color deconfinement defined here may occur at $T \lesssim T_{\mathrm{SF}}$ (see also Fig. 10 in Ref. [30] and Fig. 2 in Ref. [46] based on the Polyakov loop). Combining this tendency with the insensitivity of $T_{\mathrm{SF}}$, we conjecture that the diquark gap is created not through the soft momentum exchange, which should be sensitive to the medium, but semisoft or semihard momentum transfers that are more robust to the medium effects. We guess this observation for the two-color QCD may be transferred to the three-color case.

Finally, we briefly comment on disagreement between gluon propagators based on lattices with coarser grids but bigger volumes [28] (which we used in this paper) and those based on finer grids but smaller volumes [37]. The latter simulations were done for $N_{t} \times N_{s}^{3}=(32,24,16,8) \times 32^{3}$, which are presented as $(0,188,280,560) \mathrm{MeV}$, and they found the vanishing of the string tension at $\mu_{q} \simeq 750 \mathrm{MeV}$ at the lowest temperature. But if we apply the same estimate on temperature based on $T=1 / L_{t}$, then their results at the lowest temperature are interpreted as the results at $T \simeq 140 \mathrm{MeV}$. As we discussed in this paper, gluon propagators at $T \gtrsim T_{\mathrm{SF}}$ basically follow the explanations based on a thermal quark gas. At such a high temperature, the sensitivity of various quantities to $\mu_{q}$ is 
reasonable, and we regard that the results of Refs. [28] and [37] are rather consistent.

\section{ACKNOWLEDGMENTS}

We thank the organizers of the workshop "Probing the physics of high-density and low-temperature matter with ab initio calculations in 2-color QCD" which was held in
November 2020 at Yukawa Institute of Theoretical Physics, Kyoto University, where this work was motivated. We are grateful to Professors A. Maas and J. Skullerud for kindly providing us with their lattice data in Ref. [28] and explanations concerning with finite volume effects. T. K. is supported by NSFC Grant No. 11875144.
[1] G. Baym, T. Hatsuda, T. Kojo, P. D. Powell, Y. Song, and T. Takatsuka, Rep. Prog. Phys. 81, 056902 (2018).

[2] T. Kojo, arXiv:2011.10940.

[3] M. Leonhardt, M. Pospiech, B. Schallmo, J. Braun, C. Drischler, K. Hebeler, and A. Schwenk, Phys. Rev. Lett. 125, 142502 (2020).

[4] A. Rajan, T. Gorda, S. Liuti, and K. Yagi, arXiv:1812 .01479 .

[5] K. Fukushima, T. Kojo, and W. Weise, Phys. Rev. D 102, 096017 (2020).

[6] K. Masuda, T. Hatsuda, and T. Takatsuka, Astrophys. J. 764, 12 (2013).

[7] T. Kojo, P. D. Powell, Y. Song, and G. Baym, Phys. Rev. D 91, 045003 (2015).

[8] A. Kurkela, P. Romatschke, and A. Vuorinen, Phys. Rev. D 81, 105021 (2010).

[9] A. Kurkela, E.S. Fraga, J. Schaffner-Bielich, and A. Vuorinen, Astrophys. J. 789, 127 (2014).

[10] Y. Song, G. Baym, T. Hatsuda, and T. Kojo, Phys. Rev. D 100, 034018 (2019).

[11] D. Suenaga and T. Kojo, Phys. Rev. D 100, 076017 (2019).

[12] L. von Smekal, A. Hauck, and R. Alkofer, Ann. Phys. (N.Y.) 267, 1 (1998); 269, 182(E) (1998).

[13] R. Alkofer and L. von Smekal, Phys. Rep. 353 (2001), 281.

[14] R. Alkofer, Braz. J. Phys. 37, 144 (2007).

[15] C. S. Fischer, A. Maas, and J. M. Pawlowski, Ann. Phys. (Amsterdam) 324, 2408 (2009).

[16] A. K. Cyrol, L. Fister, M. Mitter, J. M. Pawlowski, and N. Strodthoff, Phys. Rev. D 94, 054005 (2016).

[17] A. Cucchieri, D. Dudal, T. Mendes, and N. Vandersickel, Phys. Rev. D 85, 094513 (2012).

[18] A. Maas, Phys. Rev. D 91, 034502 (2015).

[19] G. Curci and R. Ferrari, Nuovo Cimento A 32, 151 (1976).

[20] M. Tissier and N. Wschebor, Phys. Rev. D 84, 045018 (2011).

[21] U. Reinosa, J. Serreau, M. Tissier, and N. Wschebor, Phys. Rev. D 96, 014005 (2017).

[22] U. Reinosa, J. Serreau, M. Tissier, and N. Wschebor, Phys. Rev. D 89, 105016 (2014).

[23] U. Reinosa, J. Serreau, M. Tissier, and A. Tresmontant, Phys. Rev. D 95, 045014 (2017).

[24] M. Peláez, M. Tissier, and N. Wschebor, Phys. Rev. D 90, 065031 (2014).

[25] J. B. Kogut, M. A. Stephanov, D. Toublan, J. J. M. Verbaarschot, and A. Zhitnitsky, Nucl. Phys. B582, 477 (2000).
[26] J. B. Kogut, M. A. Stephanov, and D. Toublan, Phys. Lett. B 464, 183 (1999).

[27] T. Boz, P. Giudice, S. Hands, and J. I. Skullerud, Phys. Rev. D 101, 074506 (2020).

[28] T. Boz, O. Hajizadeh, A. Maas, and J. I. Skullerud, Phys. Rev. D 99, 074514 (2019).

[29] T. Boz, S. Cotter, L. Fister, D. Mehta, and J. I. Skullerud, Eur. Phys. J. A 49, 87 (2013).

[30] S. Cotter, P. Giudice, S. Hands, and J. I. Skullerud, Phys. Rev. D 87, 034507 (2013).

[31] S. Hands, P. Kenny, S. Kim, and J. I. Skullerud, Eur. Phys. J. A 47, 60 (2011).

[32] S. Hands, S. Kim, and J. I. Skullerud, Phys. Rev. D 81, 091502 (2010).

[33] S. Hands, P. Sitch, and J. I. Skullerud, Phys. Lett. B 662, 405 (2008).

[34] S. Hands, S. Kim, and J. I. Skullerud, Eur. Phys. J. C 48, 193 (2006).

[35] N. Astrakhantsev, V. V. Braguta, E. M. Ilgenfritz, A. Y. Kotov, and A. A. Nikolaev, Phys. Rev. D 102, 074507 (2020).

[36] N. Y. Astrakhantsev, V. G. Bornyakov, V. V. Braguta, E. M. Ilgenfritz, A. Y. Kotov, A. A. Nikolaev, and A. Rothkopf, J. High Energy Phys. 05 (2019) 171.

[37] V. G. Bornyakov, V. V. Braguta, A. A. Nikolaev, and R. N. Rogalyov, Phys. Rev. D 102, 114511 (2020).

[38] V. G. Bornyakov, V. V. Braguta, E. M. Ilgenfritz, A. Y. Kotov, A. V. Molochkov, and A. A. Nikolaev, J. High Energy Phys. 03 (2018) 161.

[39] V. V. Braguta, E. M. Ilgenfritz, A. Y. Kotov, A. V. Molochkov, and A. A. Nikolaev, Phys. Rev. D 94, 114510 (2016).

[40] V. V. Braguta, V. A. Goy, E. M. Ilgenfritz, A. Y. Kotov, A. V. Molochkov, M. Muller-Preussker, and B. Petersson, J. High Energy Phys. 06 (2015) 094.

[41] V. Braguta, M. N. Chernodub, V. A. Goy, K. Landsteiner, A. V. Molochkov, and M. I. Polikarpov, Phys. Rev. D 89, 074510 (2014).

[42] V. Braguta, M. N. Chernodub, K. Landsteiner, M. I. Polikarpov, and M. V. Ulybyshev, Phys. Rev. D 88, 071501 (2013).

[43] P. V. Buividovich, D. Smith, and L. von Smekal, arXiv: 2012.05184; Phys. Rev. D 102, 094510 (2020).

[44] M. Puhr and P. V. Buividovich, Phys. Rev. Lett. 118, 192003 (2017). 
[45] J. Wilhelm, L. Holicki, D. Smith, B. Wellegehausen, and L. von Smekal, Phys. Rev. D 100, 114507 (2019).

[46] K. Iida, E. Itou, and T. G. Lee, J. High Energy Phys. 01 (2020) 181; Prog. Theor. Exp. Phys. 2021, 013 B05 (2021).

[47] S. Muroya, A. Nakamura, and C. Nonaka, Phys. Lett. B 551, 305 (2003).

[48] T. Makiyama, Y. Sakai, T. Saito, M. Ishii, J. Takahashi, K. Kashiwa, H. Kouno, A. Nakamura, and M. Yahiro, Phys. Rev. D 93, 014505 (2016).

[49] G. f. Sun, L. He, and P. Zhuang, Phys. Rev. D 75, 096004 (2007).

[50] L. He, Phys. Rev. D 82, 096003 (2010).

[51] T. Kanazawa, T. Wettig, and N. Yamamoto, J. High Energy Phys. 08 (2009) 003.

[52] T. Brauner, K. Fukushima, and Y. Hidaka, Phys. Rev. D 80, 074035 (2009); 81, 119904(E) (2010).

[53] J. O. Andersen and T. Brauner, Phys. Rev. D 81, 096004 (2010).

[54] J. O. Andersen, T. Brauner, and W. Naylor, Phys. Rev. D 92 , 114504 (2015).

[55] R. Contant and M. Q. Huber, Phys. Rev. D 101, 014016 (2020); 96, 074002 (2017).

[56] N. Strodthoff and L. von Smekal, Phys. Lett. B 731, 350 (2014).

[57] N. Strodthoff, B. J. Schaefer, and L. von Smekal, Phys. Rev. D 85, 074007 (2012).

[58] T. Kojo and G. Baym, Phys. Rev. D 89, 125008 (2014).

[59] D. H. Rischke, Phys. Rev. D 62, 034007 (2000).

[60] D. H. Rischke, D. T. Son, and M. A. Stephanov, Phys. Rev. Lett. 87, 062001 (2001).

[61] T. Kojo, D. Hou, J. Okafor, and H. Togashi, arXiv:2012.01650.

[62] L. McLerran and R. D. Pisarski, Nucl. Phys. A796, 83 (2007).

[63] L. McLerran and S. Reddy, Phys. Rev. Lett. 122, 122701 (2019).

[64] R. D. Pisarski, V. V. Skokov, and A. M. Tsvelik, Phys. Rev. D 99, 074025 (2019).

[65] A. Andronic, D. Blaschke, P. Braun-Munzinger, J. Cleymans, K. Fukushima, L. D. McLerran, H. Oeschler,
R. D. Pisarski, K. Redlich, C. Sasaki et al., Nucl. Phys. A837, 65 (2010).

[66] Y. Hidaka, L. D. McLerran, and R. D. Pisarski, Nucl. Phys. A808, 117 (2008).

[67] D. C. Duarte, S. Hernandez-Ortiz, and K. S. Jeong, Phys. Rev. C 102, 065202 (2020); 102, 025203 (2020).

[68] K. S. Jeong, L. McLerran, and S. Sen, Phys. Rev. C 101, 035201 (2020).

[69] T. Kojo, Nucl. Phys. A877, 70 (2012); T. Kojo, Y. Hidaka, K. Fukushima, L. D. McLerran, and R. D. Pisarski, Nucl. Phys. A875, 94 (2012); T. Kojo, R. D. Pisarski, and A. M. Tsvelik, Phys. Rev. D 82, 074015 (2010); T. Kojo, Y. Hidaka, L. McLerran, and R. D. Pisarski, Nucl. Phys. A843, 37 (2010).

[70] E. J. Ferrer, V. de la Incera, and A. Sanchez, Acta Phys. Pol. B Proc. Suppl. 5, 679 (2012).

[71] L. McLerran, K. Redlich, and C. Sasaki, Nucl. Phys. A824, 86 (2009).

[72] G. Cao and J. Liao, J. High Energy Phys. 10 (2020) 168.

[73] T. Zhao and J. M. Lattimer, Phys. Rev. D 102, 023021 (2020).

[74] S. Sen and N. C. Warrington, Nucl. Phys. A1006, 122059 (2021).

[75] R. D. Pisarski and D. H. Rischke, Phys. Rev. D 61, 051501 (2000).

[76] L. P. Gor'kov and T. M. Melik-Barkhudarov, Zh. Eksp. Teor. Fiz. 40, 1452 (1961) [Sov. Phys. JETP 13, 1018 (1961)], http://www.jetp.ac.ru/cgi-bin/e/index/e/13/5/p1018?a=list.

[77] L. Pisani, A. Perali, P. Pieri, and G. Calvanese Strinati, Phys. Rev. B 97, 014528 (2018).

[78] R. Hagedorn, Nuovo Cimento Suppl. 3, 147 (1965), http:// cds.cern.ch/record/346206? ln=ja.

[79] A. M. Polyakov, Phys. Lett. 72B, 477 (1978).

[80] T. Banks and E. Rabinovici, Nucl. Phys. B160, 349 (1979).

[81] M. Hanada, J. Maltz, and L. Susskind, Phys. Rev. D 90, 105019 (2014). 